\title{
PROCESSUS DIGESTIFS DES RUMINANTS III. - ÉTUDE IN-VITRO DE QUELQUES PHÉNOMÈNES DE DIGESTIBILITE ASSOCIATIVE
}

PAR

\author{
A. C. FRANÇOIS, ( $\left.{ }^{1}\right)$ A. M. LEROY, S. Z. ZELTER $\left({ }^{2}\right)$ \\ Laboratoire de Recherches Zootechniques, Institut national agronomique, \\ Paris
}

On sait maintenant que la production d'acides gras volatils dans l'appareil digestif constitue un aspect fondamental de la nutrition du Ruminant. Diverses mises au point ont été.publiées sur cette question au cours des dernières années (I) (2) (3).

Le taux de chacun des acides formés, notamment, acétique, propionique, butyrique, dépend dans une large mesure des glucides du régime [Marshali, et Philitipson (4), Eilsden (5), McNaught (6)]. Avec un tégime d'herbe ou de foin, on observe une prédominance de la fermentation acétique et une absence quasiment totale d'acide lactique. [Gray et coll. (7), Fauconneau et coll. (8)]. Par ailleurs, Philimipson (9), a montré que les flocons de maïs provoquaient chez l'agneau l'apparition de quantités importantes d'acide lactique, et secondairement d'acide propionique. Il en est de même pour les régimes à base de rutabaga, [PHILLIPSON et MCANALLY (IO)].

Le rôle physiologique qu'attachent de nombreux auteurs aux acides gras inférieurs, et en particulier à l'acide acétique, pour la lipogénèse mammaire, [(FOLLEX et FRENCH (II), ZELTER (I2))], nous a conduit à observer les effets des modifications de rapport entre les constituants $\mathrm{du}$ régime alimentaire du ruminant, et l'intensité de formation de cet acide au niveau du Rumen. Nous avons étudié dans ce dessein divers types d'associations alimentaires utilisés couramment dans la pratique.

\section{TECHNIQUE EXPERIMENTALE}

La technique in-vitro ainsi que les techniques analytiques utilisées ont été décrites dans un précédent mémoire (8). Nous préciserons seulement que plusieurs substrats ont été expérimentés soit isolément, soit

(') Adresse actuelle : Service de Biochimie, C. N. R. Z. Jouy en Josas.

(2) Avec la collaboration technique de Mme Navil.te et MIIe Dumay.

Annales de Zootechnie. - 1955 
en association et en proportions variables, le foin de luzerne servant de substrat-type. Ont été étudiés : foin de luzerne, pomme de terre crue, pomme de terre cuite, betterave demi-sucrière, mélasse de betterave; des glucides purs tels que : 1'amidon, le glucose, le saccharose, le lactose, la cellulose (coton linters) ; des fourrages verts : fléole, ray-grass, luzerne, trèfle du Poitou, trèfle violet breton.

Une quantité totale d'environ $25 \mathrm{~g}$ de matière sèche de substrat était généralement utilisée à l'exception des glucides purs introduits à la dose de Io g. Les critères de comparaison étaient d'une part le taux de disparition des glucides (cellulose et glucides réducteurs après hydrolyse) et d'autre part l'apparition d'acides gras volatils (acétique, propionique, butyrique), d'acide lactique, et la valeur du $\mathrm{pH}$.

\section{RESULTATS}

Les résultats sont consignés dans les tableaux I-A, I-B et II ci-après.

\section{INTERPRETATION ET DISCUSSION}

\section{A) Fourrages verts}

La dégradation des fourrages verts au niveau du rumen (tableau I-A) est caractérisée par l'absence totale d'acide lactique. A poids égal de substrat sec, les quantités totales d'acides volatils formés semblent plus élevées pour les légumineuses que pour les graminées. Toutefois, les répartitions en acides acétique, propionique et butyrique, ne diffèrent guère entre les deux familles. L'apparition de quantités plus élevées d'acides volatils engendrés par les légumineuses, malgré la disparition de quantités plus faibles de glucides, peut résulter de la désamination des protides, [E, $\mathrm{EchazL}_{4}$ ( $\left.(\mathrm{I} 3)\right]$. En effet, les teneurs en azote total pour Ioo parties de matière sèche de substrat étaient les suivantes : fléole : I.19, ray-grass : 1.54 , luzerne. 4.69 , trèfle du Poitou : 3.43 , trèfle violet breton : 4.09 .

\section{B) Glucides purs}

Le faciès du processus fermentaire est quelque peu différent entre les essais $\mathrm{A}$ et $\mathrm{B}$; ceci peut être attribué à la provenance différente des inoculums utilisés.

Nos résultats confirment que ni l'amidon, ni la cellulose ne conduisent à une fermentation lactique (tableau I-B). En revanche, les sucres solubles (saccharose, lactose, glucose) sont essentiellement générateurs 
d'acide lactique. Les quantités d'acides volatils apparues sont du même ordre de grandeur quel que soit 1e substrat glucidique, bien que le taux de disparition des glucides réducteurs soit plus élevé dans le cas des sucres solubles. Les données montrent que les valeurs des $\mathrm{pH}$ sont nettement plus basses avec ces derniers substrats qu'avec l'amidon ou la cellulose, et que la fermentation propionique prédomine dans le cas de la cellulose. Cette dernière observation est en accord avec celle de ErSDEN (5) et de MaRston (I4).

\section{C) Associations alimentaires}

Notons tout d'abord que pour la pomme de terre l'aspect de la fermentation diffère sensiblement selon son état. Introduite seule à l'état cuit, elle conduit à une fermentation à prédominance butyrique, tandis qu'à l'état cru, l'acide acétique prédomine. Parallèlement, il y a dans ce dernier cas formation d'acide lactique qui n'apparaît pas dans l'autre. Le type de fermentation de la pomme de terre crue est à rapprocher de celui de 1'amidon pur (tableau I-A).

Dans le cas du foin seul, la fermentation présente l'allure connue caractérisée par l'absence totale d'acide lactique et la prédominance d'acides acétique. En introduisant dans le milieu des proportions croissantes de pomme de terre cuite, aux dépens du foin de luzerne, on constate une régression continue de la fermentation acétique et un accroissement parallèle de l'acide butyrique, tandis que le propionique ne varie que très faiblement. Ces déviations ne s'observent pas pour les pommes de terre à l'état cru. Pour faire dévier la fermentation du type "foin " vers celle qui caractérise la pomme de terre, un certain rapport semble devoir être réalisé entre les quantités de matière sèche associées de ces deux aliments.

Du point de vue de la physiologie de la rutrition du ruminant, on peut se demander si la cuisson de la pomme de terre, qui aboutit à une dépression de la fermentation acétique, est souhaitable. Toutefois, l'effet associatif du foin de luzerne peut atténuer cet inconvénient dans la mesure où le rapport M. S. pomme de terre/M. S. foin reste en deça de $I / I$.

Notons que la digestibilité in-vitro des glucides de la pomme de terre est améliorée par la cuisson, et que si le rapport M. S. pomme de terre/M. S. foin dépasse I/I, l'effet associatif du premier de ces aliments se manifeste par une dépression de la digestibilité in-vitro de la cellulose. Ceci est à rapprocher des observations in-vivo signalées par les anciens auteurs (KUHN) et confirmées par SwIFT et coll. (I5) et par BUrroughs et coll. (r6).

Le comportement de la betterave demi-sucrière seule ou de la mélasse 

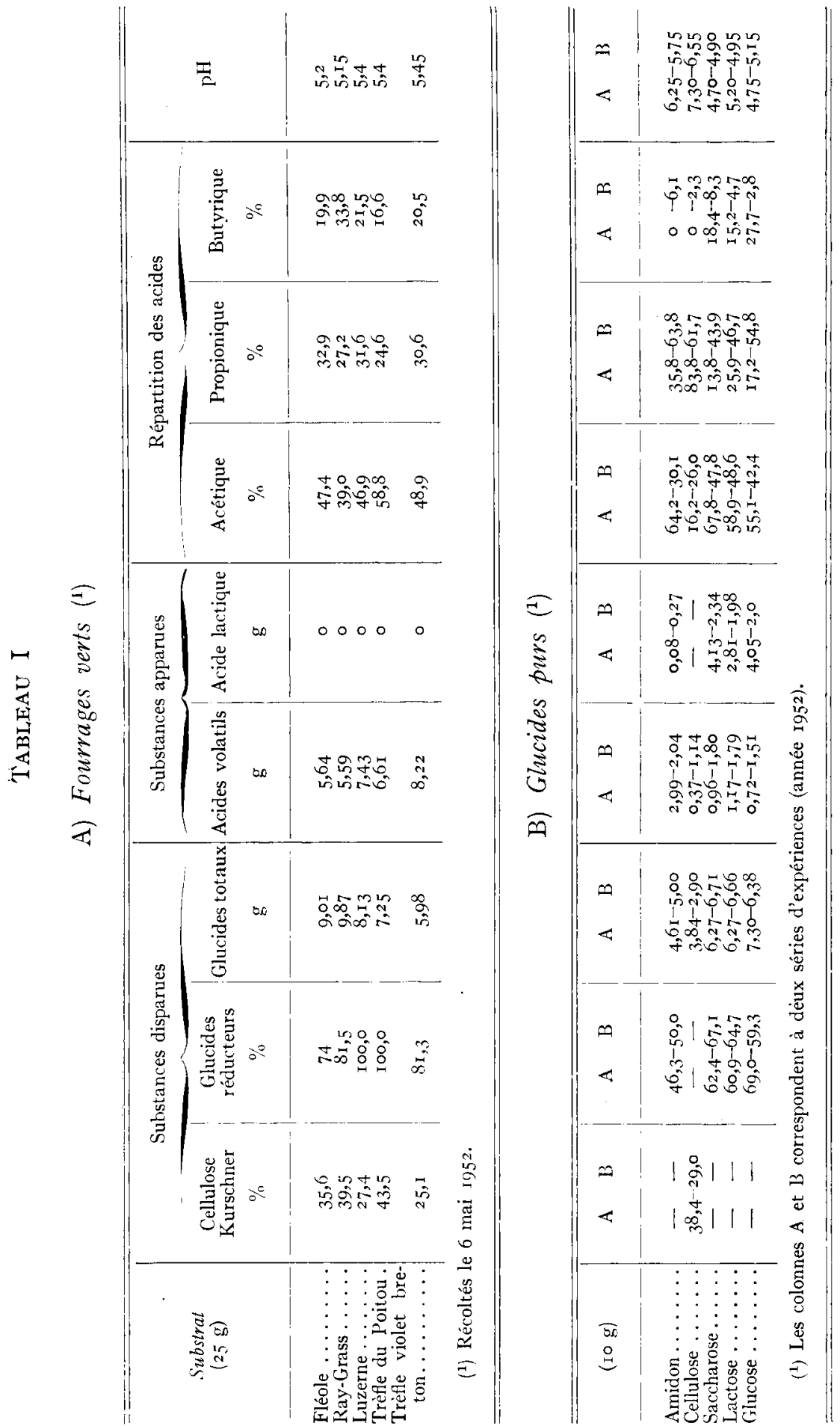
de betterave (tableau II $b$, II $c$ ) ressemble à celui du saccharose ou des autres sucres solubles (tableau II B). La fermentation lactique est de loin prédominante. L'association en proportions croissantes de matière sèche de foin de luzerne à la mélasse ou à la betterave, inverse le cours des fermentations dans le sens de l'acidité volatile. Là encore, la déviation apparaît brusquement lorsque le rapport $\frac{\text { M. S. mélasse ou betterave }}{\text { M. S. foin }}$ atteint le seuil I/I. Cette déviation s'accompagne d'une chute des taux de disparition de la cellulose. Ceci confirme également les observations faites in-vivo par LEHMANN et par KELLNER. L'ensemble de nos observations montre que la valeur du $\mathrm{pH}$ du milieu du rumen est réglée par l'intensité de la fermentation lactique, qui elle-même dépend de la composition de la ration.

ELSDEN a montré que l'aciđe lactique peut lui-même fermenter au niveau du rumen et être transformé en acides gras volatils. Toutefois, à la lumière de nos données, il apparaît que si un tel phénomène peut se produire en milieu dilué, ce n'est pas le cas lorsqu'il se forme une quantité massive d'acide lactique.

RoBINson et coll. (I7) ont, de leur côté, récemment montré l'influence de la concentration et du $\mathrm{pH}$ sur la métabolisation de l'acide lactique. A pH 5.0 et à $\mathrm{pH} \mathrm{8,} \mathrm{l'acide} \mathrm{lactique} \mathrm{s'accumule.} \mathrm{Or,} \mathrm{les} \mathrm{consommations}$ exagérées d'aliments glucidiques (betteraves notamment), peuvent conduire à un tel processus : production rapide d'acide lactique, provoquant un abaissement du $\mathrm{pH}$ qui à son tour favorise l'accumulation de l'acide lactique. Il peut en résulter des accidents digestifs et une dépression de la lipogénèse mammaire due à une formation insuffisante d'acides gras volatils.

\section{RESUME ET CONCLUSIONS}

Io Au moyen d'une technique de digestion in-vitro, la formation d'acides gras volatils et d'acide lactique à partir de divers substrats a été étudiée : l'influence de l'association d'aliments glucidiques à du foin de luzerne a été notamment suivie.

$2^{\circ}$ Les betteraves, la mélasse, le glucose, le saccharose, le lactose sont générateurs d'acide lactique. En revanche, les fourrages verts, l'amidon, la pomme de terre n'en produisent pas ou en produisent de très faibles quantités.

$3^{\circ}$ L'association du foin de luzerne aux betteraves ou à la mélasse favorise la formation d'acides volatils au détriment de l'acide lactique. Le faciès normal de la fermentation est modifié lorsque le rapport de la matière sèche de l'aliment glucidique à celle du foin est supérieur à $\mathrm{I}$. 


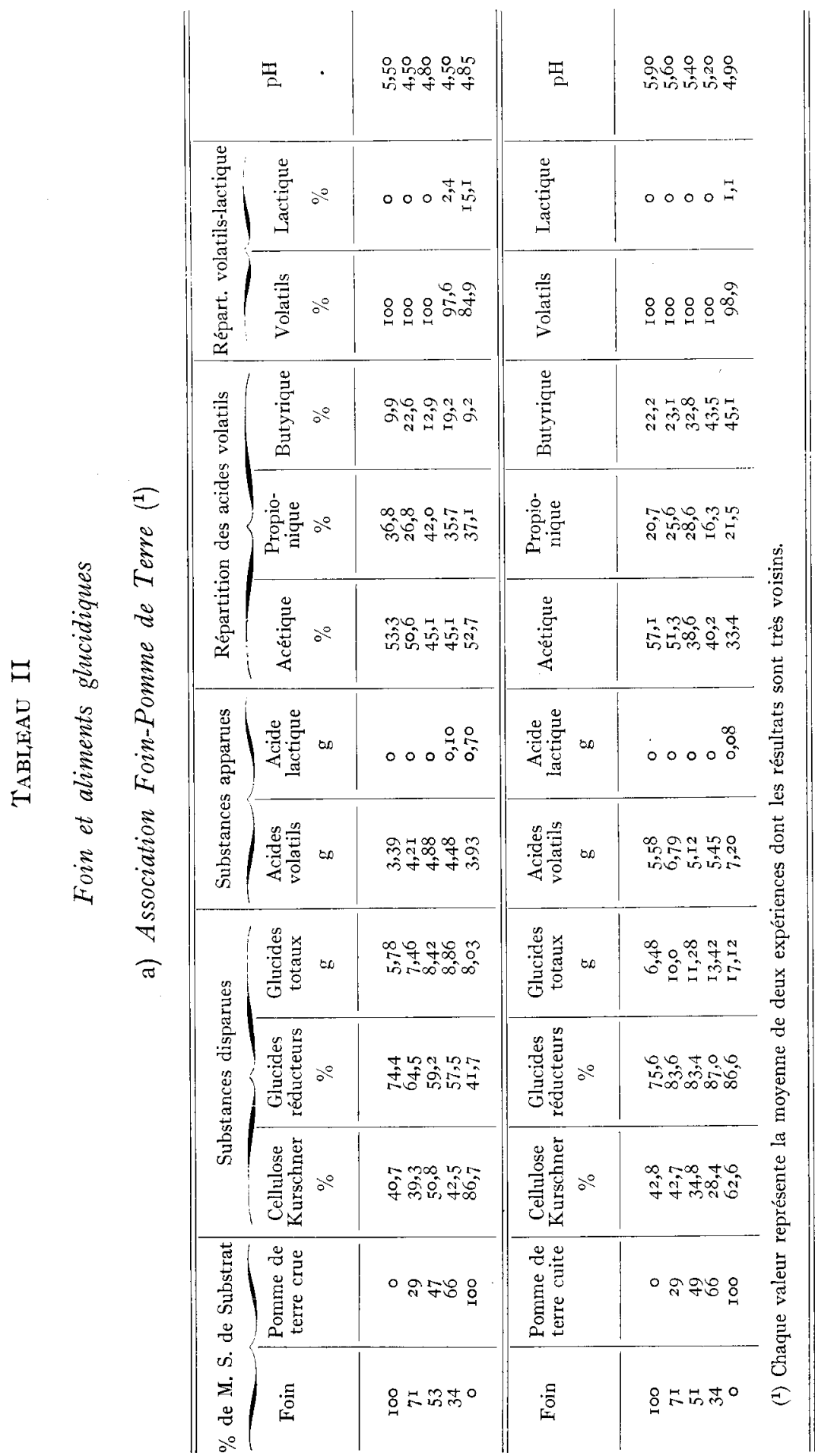




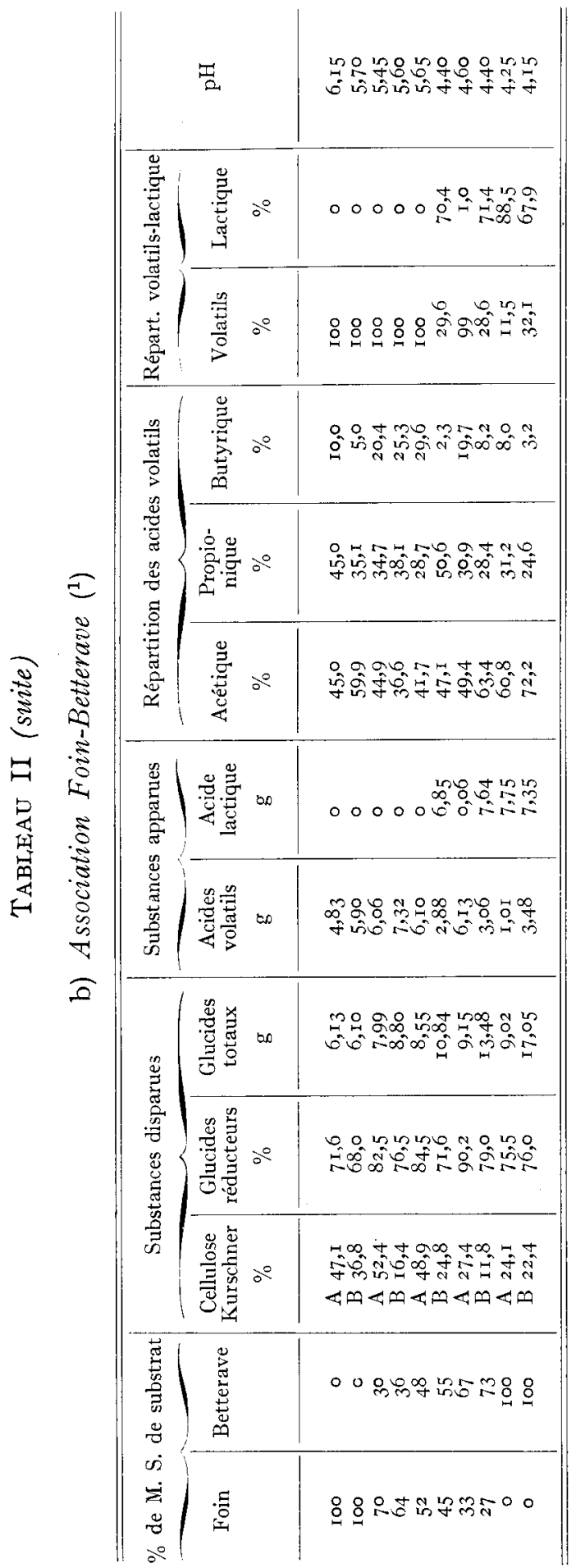

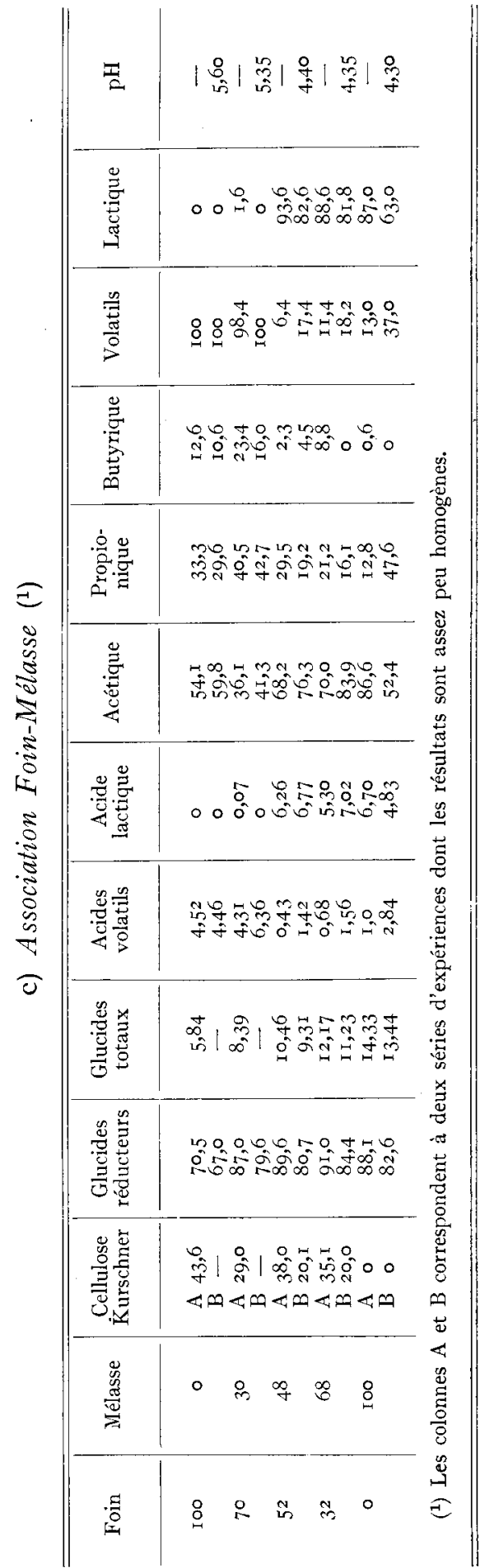




\section{RÉFÉRENCES BIBLIOGRAPHIQUES}

(I) Eisden (S. R.), Phillipson (A. T.). - Ruminant digestion. Ann. Rev. Biochem., 1\%, 705, I948.

(2) Hufrman (C. F.). - Ruminant nutrition. Ann. Rev. Biochem., 22, 339, I953.

(3) JARRIGE (R.). - L'utilisation des glucides alimentaires par les Ruminants. Ann. Nut. Alim. 7, 245 et 399, I953.

(4) Marshali (R. A.), Philitipson (A. 'T.). - Production and absorption of volatile acids from the rumen. Proc. Nut. Soc., 3, 238 , I945.

(5) EISDEN (S. R.). - The fermentation of carbohydrates in the rumen of the sheep. J. Exp. Biol., 22, 5I, I945.

(6) McNaughT (M. I.). - The utilization of non-protein nitrogen in the bovine rumen. 7 - A qualitative and quantitative study of the breakdown of carbohydrate which accompanies protein formation in bovine rumen contents during in-vitro incubations. Bioch. J., 49, 325, I95I.

(7) Gray (F. V.), Pilgrim (A. F.), Weller (A. R.). - Fermentation in the rumen of the sheep. I. - The production of volatile fatty acids and methane during the fermentation of wheaten hay and lucerne hay in-vitro by microorganisms from the rumen. J. Exp. Biol., 28, 74, I95I.

(8) Fauconneau (G.), François (A. C.), Leroy (A. M.), Zelter (S. Z.). Processus digestifs des ruminants. I. - Etude in-vitro de la digestion du foin de luzerne. Ann. de Zoot., 2, 269, I953.

(9) Phillipson (A. T.). -- The fatty acids present in the rumen of lambs fed on a flaked maize ration. Brit. J. Nutr., 6, I90, I952.

(io) Philitipson (A. T.), McAnaliy (R. A.). - Studies on the fate of carbohydrates in the rumen of the sheep. J. Exp. Biol., 19, I99, I942.

(II) FOLLEY (S. J.), FRENCH ('T. H.). - The intermediary metabolism of the mammary gland. Bioch. J., 45, I I 7, I 949.

(I2) ZEITER (S. Z.). - Le rôle nutritionnel, chez la vache en lactation, des acides acétique et butyrique formés au cours de l'ensilage. Thèse, Fac. Sc. de Paris, I953.

(I3) EI, SchazLY (K.). - Degradation of protein in the rumen of sheep. I. - Some volatile fatty acids, including branched chain isomers found in vivo. Bioch. J., 51, 640, 1952.

(I4) Marston (A. R.). - The fermentation of cellulose in-vitro by organisms from the rumen of sheep. Bioch. J., 42, 564, I 948.

(I5) Swifi (R. W.), Thacker (E. J.), Biack (A.), Bratzleer (J. W.), James (W. H.). - Digestibility of rations as affected by proportion of nutrients. J. Anim. Sci., 6, 432, I947.

(i6) Burroughs (W.), Gerlaugh (P.), Edgington (B. H.), Bethke (R. M.). - Influence of cornstarch on roughage digestion in cattle. J. Anim. Sc., 8, 27I, I949.

(I7) Robinson (R. Q.), Doetsch (R. N.), Sirotnak (F. M.), Shaw (J. C.). -Production of lactic acid and an iodine staining substance by bovine rumen bacteria. J. Dairy Sc., 38, I3, 1955. 